\title{
On the Burnside problem for Semigroups of Matrices in the $(\max ,+)$ Algebra
}

\author{
S. Gaubert
}

Communicated by J.E. Pin

\begin{abstract}
We show that the answer to the Burnside problem is positive for semigroups of matrices with entries in the (max, + )-algebra (that is, the semiring ( $\mathbb{R} \cup$ $\{-\infty\}$, max,+$)$ ), and also for semigroups of (max, + -linear projective maps with rational entries. An application to the estimation of the Lyapunov exponent of certain products of random matrices is also discussed.
\end{abstract}

\section{Introduction}

The "(max, + )-algebra" is a traditional name for the semiring $(\mathbb{R} \cup\{-\infty\}, \max ,+)$, denoted $\mathbb{R}_{\text {max }}$ in the sequel. This is a particular example of idempotent semiring (that is a semiring whose additive law satisfies $a \oplus a=a$ ), also known as dioid $[17,18,2]$. This algebraic structure has been popularized by its applications to Graph Theory and Operations Research [17, 8]. Linear operators in this algebra are central in Hamilton-Jacobi theory and in the study of exponential asymptotics [33]. The study of automata and semigroups of matrices over the analogous "tropical" semiring $(\mathbb{N} \cup\{+\infty\}$, min, + ) has been motivated by some decision problems in language theory $[40,41,43,21,20,32,27,24,25]$. From our point of view, the interest of the $(\max ,+)$ algebra arises from the study of Discrete Event Dynamic Systems [2, 12], where sequences driven by $\mathbb{R}_{\max }$-linear equations represent synchronization and saturation phenomena. An account of the related (max,+)-linear system theory can be found in [2, 7, 38]. Automata over the $(\max ,+)$ algebra also have noticeable applications to Discrete Event Systems [13, 15]. In particular, certain finiteness results for semigroups of matrices can be used to compute some asymptotic performance measures (mean-case and optimalcase Lyapunov exponents [13, 15], the latest being essentially equivalent to the classical asymptotic nondeterministic complexity [42]). Let us also mention that finitely generated semigroups of matrices are central in the study of the stability of random (max, + ) linear Discrete Event Systems [29, 30, 31].

In this paper, we first show that the answer to the Burnside problem for semigroups of matrices over $\mathbb{R}_{\max }$ is positive, which extends a theorem of Simon [40] for the tropical semiring. The main novelty by comparison with Simon's proof consists in using the (max, + )-spectral theory (analogous to the PerronFrobenius theory). A different proof based on an adaptation of a combinatorial argument of Straubing can be provided in another special class of dioids. Later, we consider semigroups of $(\max ,+)$-linear projective maps. In a previous paper [13], we showed that under certain coarse irreducibility assumptions, finitely generated semigroups of linear projective maps with rational entries are finite. Here, we 
extend this result, showing that the answer to the Burnside problem is also positive for semigroups of linear projective maps with rational entries. Contrarily to the non projective case, the rationality assumption is important: we provide a counter example which is based on a kind of irrational walk on the $(\max ,+)$ projective line. The decidability of the limitedness problem for rational series over $\mathbb{R}_{\max }$ is also obtained as an easy consequence of the decidability result of Hashiguchi $[20,21]$ for the same problem over the tropical semiring. We conclude by giving an application where these finiteness results allow a simple computation of the Lyapunov exponent of some particular $(\max ,+)$ automata.

\section{Statement of the Results}

In the sequel, $\oplus, \otimes$ will stand for the additive and multiplicative laws of a semiring, whose zero and unit are denoted respectively $\mathbb{D}$ and $\mathbb{1}$. E.g., for the $\mathbb{R}_{\max }$ semiring, $\oplus=\max , \otimes=+, \mathbb{0}=-\infty, \mathbb{1}=0$. The matrix operations induced by the semiring structure are defined and written as usual (e.g. for matrices with entries in $\left.\mathbb{R}_{\max },(A B)_{i j}=\bigoplus_{k} A_{i k} B_{k j}=\max _{k}\left(A_{i k}+B_{k j}\right)\right)$.

A semigroup $S$ is torsion if for all $s \in S$, there exist $n \in \mathbb{N}$ and $c \in$ $\mathbb{N} \backslash\{0\}$ such that $s^{n+c}=s^{n}$. The well known Burnside problem asks whether a finitely generated torsion semigroup is finite. See [9] for a survey. The answer is positive for matrices with entries in commutative rings [34, 22, 44]. It is also positive in some more exotic semirings, such as the tropical semiring ( $\mathbb{N} \cup$ $\{+\infty\}$, min, + ) (Simon [40]), the "dual" semiring $(\mathbb{N} \cup\{ \pm \infty\}, \max ,+)$ and the semiring of rational languages in a single letter $\left(\operatorname{Rat}\left(a^{*}\right), \cup,.\right)$ (Mascle [32]). We show that this property also holds in the case of $\mathbb{R}_{\max }$ :

Theorem 2.1. $\quad$ A finitely generated torsion semigroup $S \subset \mathbb{R}_{\max }^{n \times n}$ is finite.

Moreover, this result admits an effective translation:

Theorem 2.2. $\quad$ It is decidable if a finitely generated semigroup $S \subset \mathbb{R}_{\max }^{n \times n}$ is torsion.

Indeed, the proof provides an algorithm which after some reduction coincides with Simon's algorithm for the tropical semiring (whose complexity is essentially $3^{n^{2}}$ ).

The proof of Theorem 2.1 uses the spectral theory of $\mathbb{R}_{\max }$ together with the linearity of the natural order of $\mathbb{R}_{\max }$. In spite of certain generalizations of the spectral theory to other — non totally ordered - dioids [10, 14], the argument does not seem to extend easily. However, we mention in passing that there is another class of dioids for which we can prove that the answer to the Burnside problem is also positive. We shall use the algebraic order of dioids, which can be defined by

$$
a \leq b \Longleftrightarrow a \oplus b=b
$$

Theorem 2.3. $\quad$ Let $\mathcal{D}$ be a commutative dioid such that

$$
\forall x, \quad\{y \in \mathcal{D} \mid y \leq x\} \text { is finite } .
$$

Then, a finitely generated torsion semigroup $S \subset \mathcal{D}^{n \times n}$ is finite.

The proof is an adaptation to the dioid's case of a combinatorial argument of Straubing [44]. This yields another proof of Mascle's finiteness result for matrices over the semiring ( $\mathbb{N} \cup\{ \pm \infty\}$, max, + ) (but not for the tropical semiring, for which 
the algebraic order $\leq$ is the opposite of the standard one). Let us give another example of nontrivial dioid satisfying $(1)$. Let $(M,+)$ be a commutative monoid. Then, the dioid $\mathcal{P}_{f}(M)$ of finite subsets of $M$, equipped with $\cup$ (as addition) and sum of subsets (as product) satisfies the condition of Theorem 2.3.

We next consider linear projective semigroups. We define the matrix projective space as the quotient of $\mathbb{R}_{\max }^{n \times n}$ by the parallelism relation

$$
M \simeq M^{\prime} \Leftrightarrow \exists \lambda \in \mathbb{R}_{\max } \backslash\{\mathbb{O}\}, \quad M=\lambda M^{\prime} .
$$

We write $\mathbb{P R}_{\max }^{n \times n}$ for the quotient semigroup (of "linear projective maps"), and $\mathfrak{p}$ denotes the canonical morphism of multiplicative semigroups

$$
\mathfrak{p}: \mathbb{R}_{\max }^{n \times n} \rightarrow \mathbb{P} \mathbb{R}_{\max }^{n \times n}
$$

Let us introduce the subsemiring of $\mathbb{R}_{\max }: \mathbb{Q}_{\max } \stackrel{\text { def }}{=}(\mathbb{Q} \cup\{-\infty\}$, max, +$)$. We set $\mathbb{P} \mathbb{Q}_{\max }^{n \times n} \stackrel{\text { def }}{=} \mathfrak{p} \mathbb{Q}_{\max }^{n \times n}$.

Theorem 2.4. A finitely generated torsion semigroup $S \subset \mathbb{P} \mathbb{Q}_{\max }^{n \times n}$ is finite.

The restriction to linear projective maps with rational entries is essential, see $\S 10$. below.

Finally, we extend to $\mathbb{R}_{\max }$ a theorem of Hashiguchi [20] for rational series over the semiring $(\mathbb{N} \cup\{+\infty\}$, min, + ). See also Hashiguchi [21], Simon [43] and Krob [25] for a first extension to $(\mathbb{Z} \cup\{+\infty\}$, min, +$)$. Given a finite alphabet $\Sigma$, we denote by $\mathbb{R}_{\max }\langle\langle\Sigma\rangle\rangle$ the semiring of formal series with coefficients in $\mathbb{R}_{\max }$ and noncommuting indeterminates in $\Sigma$. The coefficient of a series $s$ at the word $w$ is written $\langle s, w\rangle$.

Theorem 2.5. It is decidable if a rational series $s \in \mathbb{R}_{\max }\langle\langle\Sigma\rangle\rangle$ is limited, that is, if the set of values of the coefficients of $s$,

$$
C=\left\{\langle s, w\rangle \mid w \in \Sigma^{*}\right\}
$$

is finite.

\section{Preliminary Results from the $(\max ,+)$ Matrix Theory}

We next recall the definition and basic properties of the $(\max ,+)$ spectral radius. The following result is classical. It has been proved many times with various degrees of generality $[16,8,6,10]$, [2, Th. 3.23].

Lemma 3.1. Let $A \in \mathbb{R}_{\max }^{n \times n}$. The following quantities are equal ${ }^{1}$ :

1. $\sup \left\{r \in \mathbb{R}_{\max } \mid \exists u \in \mathbb{R}_{\max }^{n} \backslash\{\mathbb{D}\}, A u=r u\right\} ;$

2. $\bigoplus_{k \in \mathbb{N}}\left(\operatorname{tr} A^{k}\right)^{\frac{1}{k}}=\bigoplus_{1 \leq k \leq n}\left(\operatorname{tr} A^{k}\right)^{\frac{1}{k}}=\bigoplus_{1 \leq k \leq n} \bigoplus_{i_{1} \ldots i_{k}}\left(A_{i_{1} i_{2}} \ldots A_{i_{k} i_{1}}\right)^{\frac{1}{k}}$.

This common value will be denoted by $\rho(A)$.

Of course, $a^{\frac{1}{k}}$ with the semiring notation of the (max, + )-algebra means $\frac{a}{k}$ in the usual algebra. In more conventional terms:

$$
\rho(A)=\max _{1 \leq k \leq n} \max _{i_{1} \ldots i_{k}} \frac{A_{i_{1} i_{2}}+\cdots+A_{i_{k} i_{1}}}{k} .
$$

In the sequel, it should be clear from the context whichever algebra is used.

The following lemma is almost obvious.

\footnotetext{
${ }^{1}$ We denote by $\mathbb{O}$ the vector with all entries equal to $\mathbb{O}$.
} 
Lemma 3.2. For all $A \in \mathbb{R}_{\max }^{n \times n}$ and $k \geq 1$ :

$$
\rho\left(A^{k}\right)=(\rho(A))^{k}
$$

Proof. $\quad \rho\left(A^{k}\right) \leq(\rho(A))^{k}$ follows immediately from Lemma 3.1,2. The converse inequality follows from Lemma 3.1,1; for if $A u=r u$, then $A^{k} u=r^{k} u$, hence $\rho\left(A^{k}\right) \geq(\rho(A))^{k}$.

The most useful result of the (max,+)-matrix theory is perhaps the following cyclicity theorem which is the exact (max, + ) counterpart of a well known asymptotic property for usual nonnegative matrices. Let us recall that a matrix $M$ is irreducible $e^{2}$ if $\forall i, j, i \neq j, \exists k \geq 1,\left(M^{k}\right)_{i j} \neq \mathbb{D}$.

Theorem 3.3. $\quad[6,10],\left[2\right.$, Th. 3.112] If $M \in \mathbb{R}_{\max }^{p \times p}$ is irreducible, then

$$
\exists N, c \geq 1, \forall n \geq N, \quad M^{n+c}=(\rho(M))^{c} M^{n} .
$$

where $\rho(M)$ denotes the spectral radius of $M$.

It is very natural to look for generalizations of this cyclicity property to finitely generated semigroups of matrices. To this end, we observe that (4) rewrites as follows in the projective linear semigroup $\mathbb{P R}_{\max }^{p \times p}$ :

$$
(\mathfrak{p} M)^{n}=(\mathfrak{p} M)^{n+c} .
$$

That is, an irreducible linear projective map is torsion. This suggests to consider finitely generated projective linear semigroups satisfying some irreducibility condition. Indeed, the extension to semigroups requires a stronger primitivity condition that we next introduce.

Given a finitely generated semigroup $S$ with generators $A_{1}, \ldots, A_{p}$, we introduce an alphabet $\Sigma=\left\{a_{1}, \ldots, a_{p}\right\}$, and note $\mu$ the unique morphism $\Sigma^{+} \rightarrow S$ such that $\forall i, \mu\left(a_{i}\right)=A_{i}$. We shall write equivalently $S=\mu\left(\Sigma^{+}\right)$or $S=$ $\left\langle A_{1}, \ldots, A_{p}\right\rangle$.

Definition 3.4. A semigroup $S=\mu\left(\Sigma^{+}\right) \subset \mathbb{R}_{\max }^{n \times n}$ is primitive if there exists an integer $N$ such that ${ }^{3}$

$$
|w| \geq N \Rightarrow \mu(w)>\mathbb{O}
$$

That is, we require every sufficiently long product of generators to have non-zero entries $^{4}$. This extends to semigroups the usual primitivity (or aperiodicity) notion ${ }^{5}$. We say that a set $S$ of matrices is projectively finite if $\mathfrak{p} S$ is finite. Then, we have the following partial extension of the cyclicity theorem 3.3, taken from [13, 15].

\footnotetext{
${ }^{2}$ The notion is borrowed to the Perron-Frobenius theory. See e.g. [35]. Note that with this definition, a (possibly zero) 1-dimensional matrix is automatically irreducible.

${ }^{3}$ For a matrix $A$, the notation $A>\mathbb{O}$ is a shorthand for $\forall i j, A_{i j}>\mathbb{O}$. The length of the word $w$ is denoted $|w|$.

${ }^{4}$ It is immediate to check that this notion is indeed independent of the choice of the (finite) set of generators. A more intrinsic characterization is provided in Remark 3.8 below.

${ }^{5} \mathrm{~A}$ matrix $A$ is primitive (in the sense of the Perron-Frobenius theory) if a power of $A$ has non-zero entries, i.e. if there is an integer $N$ such that $A^{N}>\mathbb{0}$. Therefore, a semigroup $S$ with a single generator $A$ is primitive iff $A$ is primitive in the Perron-Frobenius sense.
} 
Theorem 3.5. Let $A_{1}, \ldots, A_{p} \in \mathbb{Q}_{\max }^{n \times n}$. If $\left\langle A_{1}, \ldots, A_{p}\right\rangle$ is a primitive semigroup, then it is projectively finite.

Contrarily to Theorem 3.3 (relative to the case $p=1$ ), the theorem requires the entries to be rational. See $\S 10$. for a counter-example in the irrational case.

For the sake of completeness, we include the proof of Theorem 3.5, which exploits some bounding arguments and norm properties which will be more intensively used later on (in the study of the Burnside problem for projective linear semigroups). Let us introduce the following "norms":

$$
\|A\|=\bigoplus_{i j} A_{i j}=\sup _{i j} A_{i j}, \quad|A|_{\wedge}=\inf _{A_{i j} \neq \mathbb{0}} A_{i j},
$$

with the convention inf $\varnothing=+\infty$. Obviously,

$$
\begin{gathered}
\forall A, B \in \mathbb{R}_{\max }^{n \times n}, \quad\left\{\begin{array}{l}
\|A \oplus B\|_{1}=\|A\| \oplus\|B\|_{1}, \\
|A \oplus B|_{\wedge} \geq|A|_{\wedge} \wedge|B|_{\wedge}
\end{array}\right. \\
\forall A, B \in \mathbb{R}_{\max }^{n \times n}, \quad\left\{\begin{array}{l}
\|A B\| \leq\|A\||| B \|_{1}, \\
|A B|_{\wedge} \geq|A|_{\wedge}|B|_{\wedge} .
\end{array}\right.
\end{gathered}
$$

The proof relies on the following observation.

Lemma 3.6. Let $K \in \mathbb{N}$. The set $S$ of matrices $A \in \mathbb{Z}_{\max }^{n \times n}$ such that

$$
\frac{\|A\|}{|A|_{\wedge}} \leq K
$$

is projectively finite ${ }^{6}$.

Proof. After normalization, we may assume that $\forall A \in S \backslash\{\mathbb{O}\},|A|_{\wedge}=\mathbb{1}$. Since there is at most $(K+2)^{n^{2}}-1$ matrices $A \in \mathbb{Z}_{\max }^{n \times n}$ such that $\mathbb{1}=|A|_{\wedge}$ and $\|A\| \leq K$, the lemma is proven.

Proof of Theorem 3.5. Let $q$ be the $1 \mathrm{~cm}$ of the denominators of the entries of the matrices $A_{1}, \ldots, A_{p}$. Since $x \mapsto x^{q} \quad\left(x^{q}=x \times q\right.$ with the conventional notation) is an automorphism of $\mathbb{Q}_{\max }$ which maps all the entries to integers, we shall assume that $A_{1}, \ldots, A_{p} \in \mathbb{Z}_{\max }^{n \times n}$. Let

$$
\begin{aligned}
K^{\prime} & =\max \left(\left\|A_{1}||, \ldots,\right\| A_{p} \|\right), \\
K^{\prime \prime} & =\min \left(\left|A_{1}\right|_{\wedge}, \ldots,\left|A_{p}\right|_{\wedge}\right) .
\end{aligned}
$$

The primitivity assumption implies that for $w \in \Sigma^{+}$long enough, we have a factorization $w=$ sur with $|s|,|r|=N$ and $\mu(s), \mu(r), \mu(u)>\mathbb{D}$ ( $N$ is the "primitivity index" satisfying (5)). Then

$$
\|\mu(w)\|=\|\mu(s u r)\| \leq\|\mu(s)\|\|\mu(u)\|\|\mu(r)\| \leq\left(K^{\prime}\right)^{2 N} \mu(u)_{i j}
$$

for some indices $i j$ belonging to the argmax in $\|\mu(u)\|=\sup _{i j} \mu(u)_{i j}$. Moreover

$$
\mu(\text { sur })_{k l} \geq \mu(s)_{k i} \mu(u)_{i j} \mu(r)_{j l} \geq\left(K^{\prime \prime}\right)^{2 N} \mu(u)_{i j} .
$$

This implies that

$$
\frac{\|\mu(w)\|}{|\mu(w)|_{\wedge}} \leq\left(\frac{K^{\prime}}{K^{\prime \prime}}\right)^{2 N} .
$$

It remains to apply Lemma 3.6 to conclude.

\footnotetext{
${ }^{6}$ We denote by $\mathbb{Z}_{\max }$ the semiring $(\mathbb{Z} \cup\{-\infty\}$, max, +$)$. Recall that we use the semiring notation, so that $\frac{a}{b}$ or $a / b$ denote $a-b$.
} 
Remark 3.7. The primitivity condition only involves the boolean image of the semigroup $S$. That is, introducing the morphism $\pi$ from $\mathbb{R}_{\max }$ to the boolean semiring $\mathbb{B}=\{\mathbb{D}, \mathbb{1}\}$,

$$
\pi(x)= \begin{cases}\mathbb{1} & \text { if } x \neq \mathbb{0} \\ \mathbb{0} & \text { if } x=\mathbb{0}\end{cases}
$$

and extending canonically (componentwise) $\pi$ to a morphism $\mathbb{R}_{\max }^{n \times n} \rightarrow \mathbb{B}^{n \times n}$, we get that $S$ is primitive iff the finite semigroup $\pi(S)$ is primitive.

Remark 3.8. As a by-product of Remark 3.7, we get the following more intrinsic characterization: a semigroup $S$ is primitive iff all its matrices are primitive ${ }^{8}$. Indeed, the non trivial implication can be proved as follows. Let us assume that all the matrices of $S$ are primitive, and consider the finite semigroup $\pi(S)$, whose elements are primitive boolean matrices. Let $N$ denote the cardinal of $\pi(S)$. Each product of at least $N$ elements of $\pi(S)$ writes aeb, where $a, e, b \in \pi(S)$ and $e$ is idempotent [37, Chap. 1, Prop. 1.12]. Note that the only primitive and idempotent boolean matrix is the matrix $J: J_{i j}=\mathbb{1}, \forall i j$. Since the primitive boolean matrices $a, b$ have at least one non zero entry per row and column, we have $a J b=J$. Hence, $J$ is the boolean image of every product of at least $N$ elements of $S$, which means precisely that $S$ is primitive.

\section{Preparation}

We recall or prove some lemmas of general interest. The first one is a well known combinatorial result due to Brown [4]. We say that a semigroup $S$ is locally finite if any finitely generated subsemigroup of $S$ is finite.

Lemma 4.1. (Brown) Let $\varphi: S \rightarrow T$ be a morphism from a semigroup $S$ to a locally finite semigroup $T$. Then $S$ is locally finite iff for all idempotent $E \in T$, $\varphi^{-1}(E)$ is locally finite.

We now give some lemma specific to the dioid or (max,+) case. Reducible semigroups of matrices are defined in the following natural way:

Definition 4.2. $\quad$ Let $\mathcal{D}$ be a dioid and $S$ a subsemigroup of $\mathcal{D}^{n \times n}$. We say that $S$ is reducible if there exists a proper partition $\{1, \ldots, n\}=I \cup J$ such that

$$
\forall s \in S, \forall i \in I, \forall j \in J, \quad s_{i j}=\mathbb{O} .
$$

Let $\mu$ be a morphism $\Sigma^{+} \rightarrow \mathcal{D}^{n \times n}$, and $S=\mu\left(\Sigma^{+}\right)$. It is easily checked that $S$ is reducible iff there exists a constant permutation matrix $P$, two morphisms $\mu_{1}: \Sigma^{+} \rightarrow \mathcal{D}^{p \times p}, \mu_{2}: \Sigma^{+} \rightarrow \mathcal{D}^{q \times q}$ and a map $\phi: \Sigma^{+} \rightarrow \mathcal{D}^{p \times q}$, (with $1 \leq p<n$, $p+q=n)$, such that

$$
\forall w \in \Sigma^{+}, \quad P \mu(w) P^{-1}=\left[\begin{array}{cc}
\mu_{1}(w) & \phi(w) \\
\mathbb{O} & \mu_{2}(w)
\end{array}\right] .
$$

Moreover, this is clearly equivalent to saying that the matrix $M=\bigoplus_{a \in \Sigma} \mu(a)$ is reducible (i.e. non-irreducible). The interest of irreducible semigroups arises from the following lemma, which shows that, with respect to the Burnside problem, we may only consider irreducible semigroups.

\footnotetext{
${ }^{7}$ Which can be seen as a subsemiring of $\mathbb{R}_{\max }$.

${ }^{8}$ In the Perron-Frobenius sense, see Footnote 5.
} 
Lemma 4.3. Let $S=\mu\left(\Sigma^{+}\right)$be a reducible semigroup satisfying (11). Then $S$ is torsion (resp. finite) iff $\mu_{1}\left(\Sigma^{+}\right)$and $\mu_{2}\left(\Sigma^{+}\right)$are torsion (resp. finite).

Proof. It is clear that the condition is necessary. Conversely, an easy induction shows that

$$
\phi(w)=\bigoplus_{a \in \Sigma, u a v=w} \mu_{1}(u) \phi(a) \mu_{2}(v),
$$

where $u, v \in \Sigma^{*}$ and $\mu_{1}, \mu_{2}$ are extended to $\Sigma^{*}$ (the empty word being mapped to the identity matrix). Assuming that $\mu_{1}\left(\Sigma^{+}\right)$and $\mu_{2}\left(\Sigma^{+}\right)$are finite, (12) writes $\phi(w)$ as a sum of elements taken from the finite set $\mu_{1}\left(\Sigma^{*}\right) \phi(\Sigma) \mu_{2}\left(\Sigma^{*}\right)$; hence $\phi(w)$ can only take a finite number of values due to the idempotency of $\oplus$. This implies that $\mu\left(\Sigma^{+}\right)$is finite. An application of this last result to subsemigroups of $S$ with a single generator shows the implication for torsion semigroups.

We now prove some specific (max,+) lemma.

Lemma 4.4. Let $A \in \mathbb{R}_{\max }^{n \times n}$. The following assertions are equivalent:

1. A is torsion;

2. for all irreducible bloc ${ }^{9} B$ of $A, \rho(B)=\mathbb{1}$ or $\mathbb{O}$.

Proof. From Lemma 4.3, we may assume that $A$ is irreducible. The result follows readily from the cyclicity theorem 3.3.

Corollary 4.5. Let $A \in \mathbb{R}_{\max }^{n \times n}$. The following assertions are equivalent:

1. A is projectively torsion;

2. there exists $\lambda \in \mathbb{R}_{\max }$, such that for all irreducible bloc $B$ of $A, \rho(B)=\lambda$ or $\mathbb{0}$.

Proof. Corollary 4.5 if obtained from Lemma 4.4 by noticing that $A$ is projectively torsion iff $A^{n+c}=\lambda^{c} A^{n}$ for some $n, c>0$ and $\lambda \neq \mathbb{0}$, that is iff $\lambda^{-1} A$ is torsion.

Consider the following characterization of the $(\max ,+)$ spectral radius (cf. Lemma 3.1):

$$
\rho(A)=\bigoplus_{k \geq 1}\left(\operatorname{tr} A^{k}\right)^{\frac{1}{k}} .
$$

We next generalize this property to semigroups of matrices.

Proposition 4.6. Let $\Sigma=\left\{a_{1}, \ldots, a_{p}\right\}$, and $S=\mu\left(\Sigma^{+}\right)$for some morphism $\mu: \Sigma^{+} \rightarrow \mathbb{R}_{\max }^{n \times n}$. Let $M=\mu\left(a_{1}\right) \oplus \cdots \oplus \mu\left(a_{p}\right)$. Then

$$
\rho(M)=\bigoplus_{w \in \Sigma^{+}}(\operatorname{tr} \mu(w))^{\frac{1}{|w|}}=\bigoplus_{w \in \Sigma^{+}}(\rho(\mu(w)))^{\frac{1}{|w|}} .
$$

Moreover, the sup is attained in both summations ${ }^{10}$.

\footnotetext{
${ }^{9}$ By a repeated application of decomposition (11), a reducible matrix $A$ writes $A=P^{-1} T P$, where $T$ is an upper block-triangular matrix whose diagonal blocks $B$ are irreducible. We call such a $B$ an irreducible block of $A$. As noticed in Footnote 2, an irreducible block may be reduced to a zero 1-dimensional matrix.

${ }^{10}$ recall that $\bigoplus_{i} \alpha_{i}=\sup _{i} \alpha_{i}$.
} 
Proof. Since $\forall i, \mu\left(a_{i}\right) \leq M$, we have

$$
\forall w \in \Sigma^{+}, \mu(w) \leq M^{|w|},
$$

then

$$
\bigoplus_{w \in \Sigma^{+}}(\operatorname{tr} \mu(w))^{\frac{1}{|w|}} \leq \bigoplus_{k \geq 1}\left(\operatorname{tr} M^{k}\right)^{\frac{1}{k}}=\rho(M)
$$

by (13). Similarly,

$$
\bigoplus_{w \in \Sigma^{+}}(\rho(\mu(w)))^{\frac{1}{|w|}} \leq \bigoplus_{n \geq 1}\left(\rho\left(M^{n}\right)\right)^{\frac{1}{n}}=\rho(M)
$$

by Lemma 3.2. We now prove the converse inequalities. Let $i_{1}, \ldots, i_{k} \in\{1, \ldots, n\}$ such that

$$
\rho(M)^{k}=M_{i_{1} i_{2}} \ldots M_{i_{k} i_{1}} .
$$

Since $M=\bigoplus_{s} \mu\left(a_{s}\right)$, each entry of $M$ corresponds to some entry of one of the $\mu\left(a_{s}\right)$. Precisely, for each $l \in\{1, \ldots, k\}$, we can take $s_{l} \in\{1, \ldots, p\}$ such that $M_{i_{l} i_{l+1}}=\mu\left(a_{s_{l}}\right)_{i_{l} i_{l+1}}$ (with the convention $k+1=1$ ). Let $w=a_{s_{1}} \ldots a_{s_{k}}$. Then

$$
\begin{aligned}
\rho(\mu(w)) & \geq \operatorname{tr} \mu(w) \geq \mu(w)_{i_{1} i_{1}} \\
& \geq \mu\left(a_{s_{1}}\right)_{i_{1} i_{2}} \ldots \mu\left(a_{s_{k}}\right)_{i_{k} i_{1}}=\rho(M)^{k}
\end{aligned}
$$

which shows that the converse inequalities in (15) and (14) hold.

The following property of idempotent boolean matrices was already used by Krob [23, Prop 9.4] in a different context.

Lemma 4.7. Let $A \in \mathbb{B}^{n \times n}$ be an idempotent matrix. Then, the irreducible blocs of $A$ are either $\mathbb{D}$ or equal to some matrix $J_{k}$, where $J_{k}$ denotes the $k \times k$ matrix whose entries are all equal to $\mathbb{1}$.

Proof. Let $B$ be an irreducible $k \times k$-bloc taken from $A$. Since $A$ is idempotent, $B$ is idempotent as well, and we have $B=B^{+}$. Either $B$ is reduced to a single $\mathbb{D}$ element, either $B^{+}=J_{k}$.

The following property already appears in the theory of (classical) spectral radii of Hadamard products of nonnegative matrices (see Elsner, Johnson, Dias da Silva [11], Theorem 7).

Lemma 4.8. Let $M \in \mathbb{R}_{\max }^{n \times n}$ be irreducible. Then

$$
\rho(M)=\min _{D}\left\|D M D^{-1}\right\|
$$

where $D$ ranges the set of diagonal matrices with non $\mathbb{D}$ diagonal entries.

Proof. We get from Lemma 3.1,1 and 2 that $\rho(M)=\rho\left(D M D^{-1}\right) \leq\left\|D M D^{-1}\right\|$ for all $D$. We consider the case $n \geq 2$, then $\rho(M) \neq 0$ and by homogeneity, we may assume that $\rho(M)=\mathbb{1}$. Let $u$ be an associated eigenvector, i.e. $M u=u$. Set $D^{-1}=\operatorname{diag}\left(u_{1}, \ldots, u_{n}\right)$. Then, $\left\|D M D^{-1}\right\|=\bigoplus_{i j} u_{i}^{-1} M_{i j} u_{j}=\bigoplus_{i} u_{i}^{-1} u_{i}=\mathbb{1}$. Hence, the minimum in (16) is equal to $\mathbb{1}=\rho(M)$. 


\section{Proof of Theorem 2.2}

We show that it is decidable if a finitely generated (max, + ) semigroup of matrices is torsion. With a family of generators $A_{1}, \ldots, A_{p}$ of a semigroup $S$, we associate the matrix $M=\bigoplus_{i=1}^{p} A_{i}$.

Lemma 5.1. For $S$ to be torsion, it is necessary that $\rho(M)=\mathbb{1}$ or $\mathbb{O}$.

Proof. From Proposition 4.6, we get

$$
\rho(M)=(\rho(\mu(w)))^{\frac{1}{|w|}}
$$

for some $w \in \Sigma^{+}$. If $S$ is torsion, then $\mu(w)$ is torsion, hence by Lemma 4.4, $\rho(\mu(w))=\mathbb{1}$ or $\mathbb{0}$.

The case $\rho(M)=\mathbb{O}$ is immediate, then $S$ is nilpotent, a fortiori, torsion:

Proposition 5.2. $\quad \rho(M)=\mathbb{O}$ iff $S$ is nilpotent.

Proof. Let $n$ denote the size of $M$. If $\rho(M)=\mathbb{O}$, then $M$ has no circuits ${ }^{11}$. Since a length $n$ path of $M$ admits a subcircuit, $M$ has no length $n$ paths, hence $M^{n}=\mathbb{D}$. For all length $n$ word $w$, we have $\mu(w) \leq M^{n}=\mathbb{O}$, hence $S^{n}=\mathbb{0}$. Conversely, the existence of a circuit $\left(i_{1}, \ldots, i_{k+1}\right)$ implies that for all $m, \mathbb{O} \neq\left(M^{m k}\right)_{i_{1} i_{1}}$. Since $\mathbb{O} \neq M^{m k}=\bigoplus_{w \in \Sigma^{m k}} \mu(w), S$ contains arbitrarily long nonzero products.

When $\rho(M)=\mathbb{1}$, the following normalization is instrumental.

Definition 5.3. The semigroup $S$ is normalized if

$$
\sup \{\|A\| \mid A \in S\}=\mathbb{1} .
$$

Proposition 5.4. If $\rho(M)=\mathbb{1}$, the semigroup $S=\left\langle A_{1}, \ldots, A_{p}\right\rangle$ can be normalized by a diagonal similarity.

Proof. We first assume that $M$ is irreducible. Introduce the diagonal matrix $D$ as in the proof of Lemma 4.8. Setting $A_{i}^{\prime}=D A_{i} D^{-1}, M^{\prime}=\bigoplus_{i} A_{i}^{\prime}=D M D^{-1}$, we have

$$
\forall i, \quad\left\|A_{i}^{\prime}\right\| \leq\left\|M^{\prime}\right\|=\mathbb{1},
$$

hence, the semigroup $S^{\prime}=D S D^{-1}$ is normalized. When $M$ is reducible, we prove the result by induction on the number of irreducible blocks. Assume that

$$
M=\left[\begin{array}{cc}
M_{1} & N \\
\mathbb{D} & M_{2}
\end{array}\right],
$$

where by the induction hypothesis, $\left\|M_{2}\right\|=\rho\left(M_{2}\right)=\mathbb{1}$. (1) If the irreducible block $M_{1}$ is zero, set $D=\operatorname{diag}(\mathbb{1},\|N\| \mathrm{Id})$. (2) If $M_{1} \neq \mathbb{0}$, choose a diagonal matrix $D_{1}$ such that $\left\|D_{1} M_{1} D_{1}^{-1}\right\|=\rho\left(M_{1}\right) \leq \mathbb{1}$, and set $D=\operatorname{diag}\left(D_{1},\|N\|\left\|D_{1}\right\|\right.$ Id $)$. In both cases, $D S D^{-1}$ is normalized.

\footnotetext{
${ }^{11}$ Recall that a length $k$ path of a matrix $A$ is a family of $k+1$ indices $\left(i_{1}, \ldots, i_{k+1}\right)$ such that $A_{i_{1} i_{2}} \ldots A_{i_{k} i_{k+1}} \neq \mathbb{O}$. The weight of the path is equal to $A_{i_{1} i_{2}} \ldots A_{i_{k} i_{k+1}}$. A circuit is a path such that $i_{k+1}=i_{1}$.
} 
We denote by $\mathbb{R}_{\text {max }}^{-}$the subdioid of $\mathbb{R}_{\max }$ comprising the elements $\leq \mathbb{1}=0$. Using Proposition 5.4, since $S$ is torsion iff $S^{\prime}=D S D^{-1} \subset\left(\mathbb{R}_{\max }^{-}\right)^{n \times n}$ is torsion, we are reduced to the case of finitely generated subsemigroups of $\left(\mathbb{R}_{\max }^{-}\right)^{n \times n}$.

We next introduce some morphisms from $\mathbb{R}_{\max }$ and $\mathbb{R}_{\max }^{-}$to simpler structures. The morphism $\pi$ from $\mathbb{R}_{\max }$ to the boolean semiring $\mathbb{B}$ was already introduced in Remark 3.7. In the case of nonpositive reals, there is another useful morphism

$$
\psi: \quad \mathbb{R}_{\text {max }}^{-} \rightarrow \mathbb{B}, \quad \psi(x)= \begin{cases}\mathbb{1} & \text { if } x=\mathbb{1} \\ \mathbb{D} & \text { if } x<\mathbb{1}\end{cases}
$$

that we also extend to $\left(\mathbb{R}_{\max }^{-}\right)^{n \times n}$ (componentwise). We will use the product morphism: $\pi \times \psi:\left(\mathbb{R}_{\max }^{-}\right)^{n \times n} \rightarrow \mathbb{B}^{n \times n} \times \mathbb{B}^{n \times n}$. Finally, we introduce the following map borrowed to Simon's proof [40]

$$
\kappa: \mathbb{R}_{\text {max }}^{-} \rightarrow \mathbb{R}_{\text {max }}^{-}, \quad \kappa(x)= \begin{cases}\mathbb{1} & \text { if } x=\mathbb{1}, \\ -1 & \text { if } \mathbb{0}<x<\mathbb{1}, \\ \mathbb{0} & \text { if } x=\mathbb{0} .\end{cases}
$$

$\kappa$ is not a morphism, but we have

$$
\begin{aligned}
& \kappa(A \oplus B)=\kappa(A) \oplus \kappa(B), \\
& \kappa(\kappa(A) \otimes \kappa(B))=\kappa(A \otimes B) .
\end{aligned}
$$

Hence, $\kappa$ is a morphism from $\mathbb{R}_{\text {max }}^{-}$to the three elements dioid $\kappa\left(\mathbb{R}_{\text {max }}^{-}\right)=$ $\{\mathbb{0},-1, \mathbb{1}\}$ equipped with the two following laws $\oplus_{\kappa}$ and $\otimes_{\kappa}$ :

$$
a \oplus_{\kappa} b \stackrel{\text { def }}{=} a \oplus b, \quad a \otimes_{\kappa} b \stackrel{\text { def }}{=} \kappa(a \otimes b) .
$$

We naturally extend $\kappa$ to $\left(\mathbb{R}_{\max }^{-}\right)^{n \times n}$.

Proposition 5.5. Let $A \in\left(\mathbb{R}_{\max }^{-}\right)^{n \times n}$. Let $\imath$ denote the injection $\kappa\left(\left(\mathbb{R}_{\max }^{-}\right)^{n \times n}\right) \rightarrow\left(\mathbb{R}_{\text {max }}^{-}\right)^{n \times n}$. The following assertions are equivalent.

1. A is torsion

2. $20 \kappa(A)$ is torsion

3. for each non zero irreducible bloc $B$ of $A$, there exists a circuit of $B$ composed only of $\operatorname{arcs}^{12}$ of weight $\mathbb{1}$,

4. any block taken from $\psi(A)$ and corresponding ${ }^{13}$ to an irreducible bloc of $\pi(A)$ contains at least a circuit.

Proof. $\quad(4) \Leftrightarrow(3)$ is obvious.

$(1) \Longleftrightarrow(3)$ : This follows from Lemma 4.4 and the fact that since all the entries of $A$ are $\leq \mathbb{1}$, a circuit with weight $\mathbb{1}$ has all its entries equal to $\mathbb{1}$.

$(3) \Longleftrightarrow(2)$. Apply the previous result to $2 \circ \kappa(A)$, since the irreducible blocs and the entries equal to $\mathbb{1}$ are exactly the same for $A$ and $\imath \circ \kappa(A)$.

This yields the following extension to $\mathbb{R}_{\max }^{-}$of Simon's algorithm [40].

\footnotetext{
${ }^{12}$ An arc is a path of length 1 (the definition of paths and circuit was recalled in Footnote 11 supra).

${ }^{13}$ I.e. the two blocks are obtained by selecting the same rows and columns, respectively from $\psi(A)$ and $\pi(A)$.
} 
Algorithm 5.6. Compute the finite semigroup $\kappa(S)$ and for each $t \in \kappa(S)$, check that the matrix $A=i(t)$ satisfies property 5.5,3.

When the semigroup $S$ is primitive (Def. 3.4), we obtain a particularly simple result:

Theorem 5.7. A primitive semigroup $S=\mu\left(\Sigma^{+}\right) \subset\left(\mathbb{R}_{\max }^{-}\right)^{n \times n}$ is torsion iff there is no nilpotent matrix in $\psi S$.

One may equivalently replace "nilpotent" by "zero" in the statement of the theorem.

Proof. This follows from Proposition 5.5,4, since for all $A \in S, A$ admits a single irreducible block: there is a circuit in $\psi A$ iff $\psi A$ is non nilpotent (by Proposition 5.2).

Example 5.8. Let us consider the matrices

$$
A=\left[\begin{array}{cc}
0 & 1 \\
-1 & -1
\end{array}\right] \text { and } B=\left[\begin{array}{ll}
0 & 2 \\
0 & 0
\end{array}\right]
$$

(with $\rho(A)=0, \rho(B)=1$ ) and define $\tilde{A}=\rho(A)^{-1} A=A$,

$$
\tilde{B}=\rho(B)^{-1} B=\left[\begin{array}{rr}
-1 & 1 \\
-1 & -1
\end{array}\right] \text {. }
$$

We claim that $\tilde{S} \stackrel{\text { def }}{=}\langle\tilde{A}, \tilde{B}\rangle$ is torsion. In order to show that, we normalize $\tilde{S}$ as in Proposition 5.4. We have

$$
M=\tilde{A} \oplus \tilde{B}=\left[\begin{array}{rr}
0 & 1 \\
-1 & -1
\end{array}\right]
$$

$\rho(M)=\mathbb{1}=0$. Recall that all the columns $i$ of $M^{+} \stackrel{\text { def }}{=} M \oplus M^{2} \oplus \cdots$ such that $M_{i i}^{+}=\mathbb{1}$ are eigenvectors of $M$ for the eigenvalue $\mathbb{1}$ (see [2, 3.100]). Here,

$$
M^{+}=\operatorname{Id} \oplus M=\left[\begin{array}{rr}
0 & 1 \\
-1 & 0
\end{array}\right] \text {. }
$$

Let $D^{-1}=\operatorname{diag}\left(M_{11}^{+}, M_{21}^{+}\right)=\operatorname{diag}(0,-1)$ and set

$$
A^{\prime}=D \tilde{A} D^{-1}=\left[\begin{array}{rr}
0 & 0 \\
0 & -1
\end{array}\right], \quad B^{\prime}=D \tilde{B} D^{-1}=\left[\begin{array}{rr}
-1 & 0 \\
0 & -1
\end{array}\right]
$$

$\tilde{S}$ is torsion iff $\left\langle A^{\prime}, B^{\prime}\right\rangle$ is torsion. According to theorem 5.7, we have to check that $\psi\left\langle A^{\prime}, B^{\prime}\right\rangle$ has no nilpotent elements. But

$$
\psi\left(A^{\prime}\right)=\left[\begin{array}{ll}
\mathbb{1} & \mathbb{1} \\
\mathbb{1} & \mathbb{0}
\end{array}\right] \geq \psi\left(B^{\prime}\right)=\left[\begin{array}{ll}
\mathbb{0} & \mathbb{1} \\
\mathbb{1} & \mathbb{0}
\end{array}\right],
$$

hence we may bound from below a product of $k$ matrices $\psi\left(A^{\prime}\right)$ and $\psi\left(B^{\prime}\right)$ by $\psi\left(B^{\prime}\right)^{k}$. Since $\psi\left(B^{\prime}\right)$ is not nilpotent, this completes the verification. 


\section{Proof of Theorem 2.1}

We now prove that the answer to the Burnside problem is positive for $\mathbb{R}_{\max }^{n \times n}$. Using the normalization argument of the preceing section (Lemma 5.1, Propositions 5.2 and 5.4), it is enough to prove the result for a semigroup $S \subset\left(\mathbb{R}_{\max }^{-}\right)^{n \times n}$.

Proposition 6.1. Let $S \subset\left(\mathbb{R}_{\max }^{-}\right)^{n \times n}$ be a finitely generated semigroup. $S$ is finite iff the non $\mathbb{D}$ entries of the matrices of $S$ are bounded.

In other words, the finiteness of $S$ is equivalent to the following property

$$
\exists K \in \mathbb{R}^{-}, \quad \forall s \in S, \forall i, j, \quad s_{i j} \neq \mathbb{O} \Rightarrow K \leq s_{i j} .
$$

Proof. Condition (20) is clearly necessary. Conversely, assume that (20) holds. Let $A_{1}, \ldots, A_{p}$ be generators of $S$, and let

$$
\lambda=\sup \left\{\left(A_{k}\right)_{i j} \mid 1 \leq k \leq p, 1 \leq i, j \leq n, \quad \mathbb{O}<\left(A_{k}\right)_{i j}<\mathbb{1}\right\} .
$$

Let $w=a_{s_{1}} \ldots a_{s_{k}} \in \Sigma^{k}$. We have

$$
\mu(w)_{i j}=\left(A_{s_{1}}\right)_{i i_{2}} \ldots\left(A_{s_{k}}\right)_{i_{k} j}
$$

for some indices $i_{2}, \ldots, i_{k}$. We set $i_{1}=i, i_{k+1}=j$. Let $N(w)$ denote the maximal number of indices $l: 1 \leq l \leq k$ such that $\left(A_{s_{l}}\right)_{i_{l} i_{l+1}}<\mathbb{1}$ in a factorization of type (21). Then $\mu(w)_{i j} \leq \lambda^{N(w)}$. This implies that $\lambda^{N(w)} \geq K$ with $\lambda<\mathbb{1}$, hence $N(w)$ is bounded. Since $\mu(w)_{i j}$ is a product of $N(w)$ non- $\mathbb{1}$ factors taken from a finite set, $\mu(w)_{i j}$ can only take a finite number of values.

To show that a finitely generated torsion semigroup $S \in\left(\mathbb{R}_{\text {max }}^{-}\right)^{n \times n}$ is finite, it remains to check that the non $\mathbb{D}$ entries of the matrices of $S$ are bounded. Due to Brown's Lemma, we may assume that $\pi(S)=\{e\}$ for some idempotent matrix $e$. Moreover, due to Lemma 4.3, we may assume that $S$ is irreducible. Then, by Lemma 4.7, either $e=J_{n}$, either $e$ is the zero scalar matrix. In the latest case, $S=\{\mathbb{O}\}$ is finite. In the first case, we have necessarily

$$
\forall A \in S,\|A\|=\mathbb{1},
$$

otherwise $\mathbb{D}<\rho(A) \leq\|A\|<\mathbb{1}$ contradicts the fact that $A$ is torsion (by Lemma 4.4). The proof of Theorem 3.5 shows that there exists a finite real $K$ such that

$$
\forall A \in S, \frac{\|A\|}{|A|_{\wedge}} \leq K .
$$

It follows from (22) that $|A|_{\wedge} \geq K^{-1}$. This concludes the proof of Theorem 2.1.

\section{Proof of Theorem 2.3}

We next show that the answer to the Burnside problem is positive for semigroups of matrices over dioids $\mathcal{D}$ such that for all $x \in \mathcal{D},\{y \in \mathcal{D} \mid y \leq x\}$ is finite. We use the symmetrized version (for semirings) of the Amitsur-Levitski identity (see e.g. [5]). Let $X_{1}, \ldots, X_{p}$ be $p$ non commuting indeterminates and define

$$
\begin{aligned}
& S_{p}^{+}=\bigoplus_{\sigma \text { even }} X_{\sigma(1)} \ldots X_{\sigma(p)} \\
& S_{p}^{-}=\bigoplus_{\sigma \text { odd }} X_{\sigma(1)} \ldots X_{\sigma(p)}
\end{aligned}
$$

where the sums are taken over the even and odd permutations of $\{1, \ldots, p\}$. 
Lemma 7.1. Let $A$ be a commutative semiring. Then, the identity $S_{2 n}^{+}=S_{2 n}^{-}$ holds in $A^{n \times n}$.

This lemma can be easily deduced from the classical Amitsur-Levitski theorem by a technique of Reutenauer and Straubing [39, proof of Lemma 1], [12, Ch. 1, Prop. 2.1.5], [14, Prop. 2.2.1]. The proof of Theorem 2.3 consists in adapting the argument of Straubing [44]. Indeed, let $\mu$ be a morphism $\Sigma^{+} \rightarrow \mathcal{D}^{n \times n}$, such that $S=\mu\left(\Sigma^{+}\right)$is torsion. Recall that a word $w$ is $r$-divided if it admits a factorization $w=w_{1} \ldots w_{r}$ such that for all permutation $\sigma \neq \mathrm{Id}$,

$$
w_{\sigma(1)} \ldots w_{\sigma(r)}<_{\operatorname{lex}} w
$$

$\left(<_{\text {lex }}\right.$ denotes the lexicographic order). Then, Shirshov's Lemma [28, Th. 7.1.5] states that if $q \geq 2 r$, there exists an integer $N(|\Sigma|, q, r)$ such that, for all word $w \in \Sigma^{+}$such that $|w| \geq N(|\Sigma|, q, r)$, either $w$ admits a factorization $w=u v^{q} s$ with $1 \leq|v|<r$, either $w$ contains a $r$-divided factor (i.e. $w=u v s$ where $v$ is $r$-divided). Set $r=2 n, p=\max \{\#\langle\mu(w)\rangle+1|| w \mid<r\}(\#\langle\mu(w)\rangle$ is the finite order of the cyclic semigroup $\left.\left\{\mu(w), \mu\left(w^{2}\right), \ldots,\right\}\right)$, and $q=\max (p, 2 r)$. We claim that

$$
\forall w \in \Sigma^{+}, \quad \mu(w) \leq \bigoplus_{|v|<N(|\Sigma|, q, r)} \mu(v)
$$

We show this assertion by induction on $w$ with respect to the military order $\leq_{\mathrm{m}}$, $\left(u \leq_{\mathrm{m}} v\right.$ iff $|u|<|v|$ or $\left.|u|=|v|, u \leq_{\operatorname{lex}} v\right)$. Let $w$ such that $|w| \geq N(|\Sigma|, q, r)$. If $w=u v^{q} s$, then $\mu\left(v^{q}\right)=\mu\left(v^{t}\right)$ for some $t<s$, hence $\mu(w)=\mu\left(w^{\prime}\right)$ with $w^{\prime}=u v^{t} s<_{\mathrm{m}} w$, and by the induction hypothesis, we are done. Otherwise, we have $w=u v s$, where $v=v_{1} \ldots v_{r}$ is $r$-divided. The symmetrized polynomial identity (7.1) implies that

$$
\mu(v) \leq \bigoplus_{\sigma \text { odd }} \mu\left(v_{\sigma(1)} \ldots v_{\sigma(r)}\right)
$$

therefore

$$
\mu(w) \leq \bigoplus_{w^{\prime}<\mathrm{m} w} \mu\left(w^{\prime}\right)
$$

This shows (23). Thus $S=\mu\left(\Sigma^{+}\right)$is bounded above and (1) implies that it is finite.

\section{The Burnside Problem for Projective Linear Semigroups}

We show that a finitely generated torsion subsemigroup of $\mathbb{P} \mathbb{Q}_{\max }^{n \times n}$ is finite (Theorem 2.4). The particular case of primitive linear projective semigroups (see Theorem 3.5) suggests that the following quantity will play an important role.

Definition 8.1. The projective width of a set $S \subset \mathbb{R}_{\max }^{n \times n}$ is by definition

$$
\Delta(S)=\sup _{s \in S} \frac{\|s\|}{|s|_{\wedge}} .
$$

Rephrasing Lemma 3.6, we can state:

Lemma 8.2. A finitely generated semigroup $S \subset \mathbb{Q}_{\max }^{n \times n}$ is projectively finite iff its projective width $\Delta(S)$ is finite.

The key point in the proof that $\Delta(S)$ is finite is the following. 
Lemma 8.3. Let $S=\mu\left(\Sigma^{+}\right)$be a reducible projectively torsion semigroup such that $\pi(S)=\{e\}$ for some idempotent matrix $e$, take $\mu_{1}, \mu_{2}, \phi$ as in (11), and assume that $\Delta\left(\mu_{1}\left(\Sigma^{+}\right)\right)$and $\Delta\left(\mu_{2}\left(\Sigma^{+}\right)\right)$are finite. Then $\Delta\left(\mu\left(\Sigma^{+}\right)\right)$is finite.

Indeed, let us assume that Lemma 8.3 is proved. Then, using Brown's Lemma, it is enough to prove Theorem 2.4 when $\pi(S)=\{e\}$ for some boolean idempotent matrix $e \in \mathbb{B}^{n \times n}$ having the form of Lemma 4.7. We prove that $\Delta(S)$ is finite by induction on the number of irreducible blocks of $e$. For a single irreducible block: either $e=J_{n}$ for some $n$, then $S$ is primitive, and the finiteness of $\Delta(S)$ follows from the bound (9); either $e=\mathbb{D}$, and $S=\{\mathbb{O}\}$. In both cases, $S$ is projectively finite. Lemma 8.3 gives precisely the induction step, from which Theorem 2.4 follows.

It remains to show Lemma 8.3. We have to bound the different terms of the form $s_{i j} / s_{k l}$ appearing in $\|s\| /|s|_{\wedge}$. We will show that $\forall w \in \Sigma^{+}, \forall i, j \in$ $\{1,2\}, i \neq j$,

$$
\begin{aligned}
\left\|\mu_{i}(w)\right\| /\left|\mu_{j}(w)\right|_{\wedge} & \leq k \\
\|\phi(w)\| /\left|\mu_{i}(w)\right|_{\wedge} & \leq k_{i}^{\prime} \\
\left\|\mu_{i}(w)\right\| /|\phi(w)|_{\wedge} & \leq k_{i}^{\prime \prime} \\
\|\phi(w)\| /|\phi(w)|_{\wedge} & \leq k^{\prime \prime \prime}
\end{aligned}
$$

for some effective finite constants $k, k_{i}^{\prime}, k_{i}^{\prime \prime}, k^{\prime \prime \prime}$.

Let $e_{1}, e_{2}$ denote the diagonal blocks of $e$ corresponding to the partition (11). For all $w \in \Sigma^{+}$, for all $i=1,2, e_{i}=\pi\left(\mu_{i}(w)\right)$. From Lemma 4.7, we get the following alternative: A1) either $e_{i}=\mathbb{O}$ and $\left.\mu_{i}=\mathbb{O}, \mathrm{A} 2\right)$ either $e_{i}$ contains a nonzero irreducible block, and thus $\forall w \in \Sigma^{+}, \rho\left(\mu_{i}(w)\right) \neq \mathbb{D}$.

Let us check item (25). If $\mu_{i}$ or $\mu_{j}$ are zero, then for all $w \in \Sigma^{+}$, ||$\mu_{i}(w) \| /\left|\mu_{j}(w)\right|_{\wedge}=\mathbb{O}$. We may therefore assume that $\mu_{i}, \mu_{j} \neq \mathbb{0}$. Since $\mu(w)$ is projectively torsion, Corollary 4.5 together with item A2) of the alternative implies that $\rho\left(\mu_{i}(w)\right)=\rho\left(\mu_{j}(w)\right) \neq \mathbb{0}$. Noting that

$$
\left|\mu_{i}(w)\right|_{\wedge} \leq \rho\left(\mu_{i}(w)\right)=\rho\left(\mu_{j}(w)\right) \leq\left\|\mu_{j}(w)\right\|,
$$

we get

$$
\begin{aligned}
\frac{\left\|\mu_{i}(w)\right\|}{\left|\mu_{j}(w)\right|_{\curlywedge}} & \leq \frac{\left\|\mu_{i}(w)\right\|}{\left|\mu_{i}(w)\right|_{\wedge}} \frac{\left|\mu_{i}(w)\right|_{\wedge}}{\left\|\mu_{j}(w)\right\|} \frac{\left\|\mu_{j}(w)\right\|}{\left|\mu_{j}(w)\right|_{\wedge}} \\
& \leq k \stackrel{\text { def }}{=} \Delta\left(\mu_{i}\left(\Sigma^{+}\right)\right) \Delta\left(\mu_{j}\left(\Sigma^{+}\right)\right) .
\end{aligned}
$$

We next prove (26) when $i=1$. We assume that $\mu_{1} \neq \mathbb{D}$ (otherwise, there is nothing to prove). From formula (12) together with (6) we have $\|\phi(w)\|=$ $\left\|\mu_{1}(u) \phi(a) \mu_{2}(v)\right\|$ for some factorization $w=u a v$ (with $a \in \Sigma, u, v \in \Sigma^{*}$ ), hence,

$$
\begin{aligned}
\frac{\|\phi(w)\|}{\left|\mu_{1}(w)\right|_{\curlywedge}} & \leq \frac{\left\|\mu_{1}(u)|||\phi(a)||| \mu_{2}(v)\right\|}{\left|\mu_{1}(w)\right|_{\wedge}} \\
& \leq \frac{\left\|\mu _ { 1 } ( u ) \left|\left\|\phi(a)\left|\| \mu_{2}(v)\right|\right.\right.\right.}{\left|\mu_{1}(u)\right|_{\wedge}\left|\mu_{1}(a)\right|_{\wedge}\left|\mu_{1}(v)\right|_{\wedge}}(\text { by }(7)) \\
& \leq k_{1}^{\prime} \stackrel{\text { def }}{=} \Delta\left(\mu_{1}\left(\Sigma^{+}\right)\right)\left(\bigoplus_{a \in \Sigma} \frac{\|\phi(a)\|}{\left|\mu_{1}(a)\right|_{\wedge}}\right) k .
\end{aligned}
$$

The bound $k_{2}^{\prime}$ is obtained dually (replacing 1 by 2 in (30)). 
The proof of $(27)$ is similar, with $k_{i}^{\prime \prime}=\Delta\left(\mu_{i}\left(\Sigma^{+}\right)\right)\left(\bigoplus_{a \in \Sigma} \frac{\left\|\mu_{i}(a)\right\|}{|\phi(a)|_{\wedge}}\right) k$.

Finally, we prove (28). If both $\mu_{1}$ and $\mu_{2}$ are zero, then the idempotency of $e$ implies that $\phi=\mathbb{O}$, and there is nothing to prove. Otherwise, we choose $i$ such that $\mu_{i}$ is non zero. Then, the alternative implies that for all $w,\left|\mu_{i}(w)\right|_{\wedge}$ is finite, and

$$
\frac{\|\phi(w)\|}{|\phi(w)|_{\wedge}} \leq \frac{\|\phi(w)\|}{\left|\mu_{i}(w)\right|_{\wedge}} \frac{\left|\mu_{i}(w)\right|_{\wedge}}{\left\|\mu_{i}(w)\right\|} \frac{\left\|\mu_{i}(w)\right\|}{|\phi(w)|_{\wedge}} \leq k_{i}^{\prime} k_{i}^{\prime \prime} \leq k^{\prime \prime \prime} \stackrel{\text { def }}{=} \bigoplus_{j=1,2} k_{j}^{\prime} k_{j}^{\prime \prime}
$$

This concludes the proof of Lemma 8.3 and of Theorem 2.4.

\section{Decidability of the Limitedness Problem}

We now prove Theorem 2.5.

Lemma 9.1. Let $s$ be a series with trim $^{14}$ linear representation $(\alpha, \mu, \beta)$ and let $M=\bigoplus_{a \in \Sigma} \mu(a)$. If $s$ is limited, then $\rho(M)=\mathbb{1}$ or $\mathbb{D}$.

Proof. Let us assume that $\rho(M) \neq \mathbb{0}$. From Lemma 3.1,2 and Proposition 4.6 there exist $i$ and $k$ and a word $w$ (of length $k$ ) such that $\rho(M)^{k}=\left(M^{k}\right)_{i i}=$ $\mu(w)_{i i} \neq \mathbb{D}$. Since the linear representation is trim, there exist two indices $l, j$ and two words $u, v$ such that

$$
\alpha_{j} \mu(u)_{j i} \mu(w)_{i i} \mu(v)_{i l} \beta_{l} \neq \mathbb{0} .
$$

Hence, for all $p \geq 1$,

$$
\mathbb{0}<K \rho(M)^{p k} \leq\left\langle s, u w^{p} v\right\rangle
$$

for some (finite) constant $K$. Moreover,

$$
\left\langle s, u w^{p} v\right\rangle \leq\|\alpha\|\left\|M^{|u|+|v|+p k}\right\|\|\beta\| \leq K^{\prime} \rho(M)^{|u|+|v|+p k}
$$

for another (finite) constant $K^{\prime}$. If $\mathbb{D}<\rho(M)<\mathbb{1}$, (32) and (31) imply that $\left\langle s, u w^{p} v\right\rangle$ takes arbitrarily small but non- $\mathbb{D}$ values. If $\rho(M)>\mathbb{1},(31)$ shows that $\left\langle s, u w^{p} v\right\rangle$ takes arbitrarily large values. Therefore, $\rho(M) \notin\{0, \mathbb{1}\}$ contradicts the limitation of $s$.

Since a rational series $s \in \mathbb{R}_{\max }\langle\langle\Sigma\rangle\rangle$ admits a trim linear representation, by Lemma 9.1, we may perform the same reduction as in the decision algorithm for the torsion property (Propositions 5.2 and 5.4). Therefore, we are reduced to the case where

$$
\mu\left(\Sigma^{+}\right) \subset\left(\mathbb{R}_{\max }^{-}\right)^{n \times n}
$$

(but $\alpha$ and $\beta$ can have positive entries).

The conclusion is an immediate consequence of the following lemma.

Lemma 9.2. Assume that (33) holds, then $s$ is limited iff the non $\mathbb{D}$ values of $\langle s, w\rangle$ are bounded below.

That is, we require that

$$
\exists K \neq \mathbb{O}, \quad\langle s, w\rangle \neq \mathbb{O} \Rightarrow\langle s, w\rangle \geq K .
$$

Proof. This is a straightforward variant of the proof of Proposition 6.1.

It remains to show that the boundedness property (34) is decidable.

\footnotetext{
${ }^{14}$ Recall that a ( $n$-dimensional) linear representation of a series $s$ is a triple $(\alpha, \mu, \beta)$, where $\alpha \in \mathbb{R}_{\max }^{1 \times n}, \mu$ is a morphism: $\Sigma^{*} \rightarrow \mathbb{R}_{\max }^{n \times n}, \beta \in \mathbb{R}_{\max }^{n \times 1}$, and $\forall w \in \Sigma^{*},\langle s, w\rangle=\alpha \mu(w) \beta$. The representation is trim if each state is both accessible and co-accessible, i.e. $\forall i, \exists u, v \in \Sigma^{*}$, $(\alpha \mu(u))_{i} \neq \mathbb{O},(\mu(v) \beta)_{i} \neq \mathbb{0}$.
} 
Lemma 9.3. Under the assumption (33), the boundedness property (34) holds for $s$ iff it holds for the series $s^{\prime}$ given by the linear representation ${ }^{15}(\pi \alpha, \imath \kappa \mu, \pi \beta)$.

Proof. There exist two positive constants $K$ and $K^{\prime}$ such that

$$
\forall i j, \forall a \in \Sigma, \quad K \times(\imath \kappa \mu(a))_{i j} \leq \mu(a)_{i j} \leq K^{\prime} \times(\imath \kappa \mu(a))_{i j} .
$$

Noting that for $K>0, x \mapsto x^{K}=K \times x$ is an automorphism of $\mathbb{R}_{\max }$, we get that (35) is valid not only for $a \in \Sigma$, but also for $w \in \Sigma^{*}$. Therefore,

$$
K \times\left\langle s^{\prime}, w\right\rangle+|\alpha|_{\wedge}+|\beta|_{\wedge} \leq\langle s, w\rangle \leq K^{\prime} \times\left\langle s^{\prime}, w\right\rangle+\|\alpha\|+\|\beta\| .
$$

Since the representation of $s^{\prime}$ lives in $\left(\mathbb{N}^{-} \cup\{-\infty\}\right.$, max, + ), which is isomorphic to the tropical semiring, the decidability of the limitedness problem for the series $s^{\prime}$ follows from Hashiguchi's theorem [20], see also [21, 41, 43, 27]). This concludes the proof of Theorem 2.5.

\section{Counter Example}

We show that the theorem of finiteness of primitive linear projective semigroups with rational entries (Theorem 3.5) does not extend to the irrational case. A similar counter-example has been obtained independently by Mairesse [31].

Let

$$
A=\left[\begin{array}{cc}
\sqrt{2} & \eta \\
\eta & 0
\end{array}\right], B=\left[\begin{array}{ll}
0 & \eta \\
\eta & 1
\end{array}\right]
$$

where $\eta$ is a parameter to be fixed soon and $\sqrt{2}=1.414 \ldots$ We have $A, B>\mathbb{O}$. Hence, $\langle A, B\rangle$ is primitive. We claim that $\mathfrak{p}\langle A, B\rangle$ is infinite. This can be rephrased in terms of $(\max ,+)$ homographic functions. More precisely, we set for $x \in \mathbb{R}$,

$$
u(x) \stackrel{\text { def }}{=} \mathfrak{p}\left[\begin{array}{ll}
x & 0
\end{array}\right] .
$$

We have

$$
u(x) \mathfrak{p} A=u\left(h_{A}(x)\right)
$$

where the homographic map associated with $A$ is

$$
h_{A}(x) \stackrel{\text { def }}{=} \frac{\sqrt{2} x \oplus \eta}{\eta x \oplus 0} .
$$

Similarly, we have

$$
h_{B}(x) \stackrel{\text { def }}{=} \frac{x \oplus \eta}{\eta x \oplus 1}, u(x) \mathfrak{p} B=u\left(h_{B}(x)\right)
$$

Clearly, it is enough to show that the semigroup $\left\langle h_{A}, h_{B}\right\rangle$ (equipped with the composition product) is infinite. We assume that $\eta<0$, and note that

$$
\eta \leq x \leq-\eta \Rightarrow h_{A}(x)=\sqrt{2}+x, h_{B}(x)=x-1 .
$$

We choose $\eta$ such that the diameter of $[\eta,-\eta]$ is greater than $\sqrt{2}+1$. Then, for all $x \in[\eta,-\eta]$, we have $x+\sqrt{2} \in[\eta,-\eta]$ or $x-1 \in[\eta,-\eta]$. Hence, it is possible to define a sequence $x_{n} \in[\eta,-\eta]$ such that $x_{0}=0$ and $x_{n}=h_{A}\left(x_{n-1}\right)$ or $h_{B}\left(x_{n-1}\right)$. Such a "walk" in $[\eta,-\eta]$ is visualized on Figure 1. The trajectory shown corresponds to the particular choice: $x_{1}=h_{A}\left(x_{0}\right)=x_{0}+\sqrt{2}, x_{2}=h_{A}\left(x_{1}\right)$, 


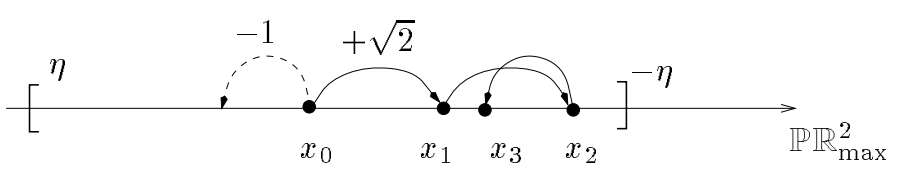

Figure 1: The $x_{n}$ sequence

$x_{3}=h_{B}\left(x_{2}\right)=x_{2}-1$. Let $p(A, k)$ (resp. $p(B, k)$ ) denote the number of choices of $h_{A}$ (resp. $\left.h_{B}\right)$ up to step $k$ (e.g., for the walk of the picture, $p(A, 3)=2, p(B, 3)=$ 1). We have

$$
\forall k \geq 0, x_{k}=p(A, k) \times \sqrt{2}-p(B, k) \times 1 .
$$

Since $p(A, k)+p(B, k)=k \rightarrow \infty$ and $1, \sqrt{2}$ are rationally independent, $x_{k}$ takes an infinite number of values. But $x_{0}$ is mapped to $x_{k}$ by an element of $\left\langle h_{A}, h_{B}\right\rangle$, and so $\left\langle h_{A}, h_{B}\right\rangle$ (hence $\mathfrak{p}\langle A, B\rangle$ ) is infinite.

The reader is referred to Mairesse [30, 31] for a complete study of the "geometry" of (max, + ) linear projective maps in small dimension (set of fixed points and attracting domains).

\section{Application: an Upper Bound for the Lyapunov Exponent}

We consider a random walk $w_{k} \in \Sigma^{k}$. That is, the word $w_{k}=a_{1} \ldots a_{k}$ (with $\left.a_{i} \in \Sigma\right)$ occurs with the probability $p\left(w_{k}\right)=p\left(a_{1}\right) \ldots p\left(a_{k}\right)$ where the $p(a)$ are given nonnegative numbers such that $\sum_{a \in \Sigma} p(a)=1$. Let $\mu: \Sigma^{+} \rightarrow \mathbb{R}_{\max }^{p \times p}$ be a morphism. The $(\max ,+)$ Lyapunov exponent $\ell$ of $\mu$ measures the growth of a random product of $k$ matrices:

$$
\ell \stackrel{\text { def }}{=} \lim _{k} \mathbb{E}\left(\left\|\mu\left(w_{k}\right)\right\|\right)^{\frac{1}{k}}=\lim _{k}\left(\left\|\mu\left(w_{k}\right)\right\|\right)^{\frac{1}{k}} \text { a.s. }
$$

This (max, +) Lyapunov exponent was introduced by Baccelli, by analogy with the maximal Lyapunov exponents of random products of matrices in the conventional algebra. See $[2,3,29,30,31]$ for a complete and more general presentation (in terms of random products). See also $[13,15]$.

As a by-product of the above characterizations of finite semigroups, we obtain an upper-bound for the Lyapunov exponent, together with an equality case.

Theorem 11.1. Let $\mu: \Sigma^{+} \rightarrow \mathbb{R}_{\max }^{n \times n}$ such that $\forall a \in \Sigma, \rho(\mu(a)) \neq \mathbb{0}$ and define the morphism:

$$
\tilde{\mu}: \forall a \in \Sigma, \tilde{\mu}(a) \stackrel{\text { def }}{=} \rho(\mu(a))^{-1} \mu(a)
$$

with the associated "worst case matrix"

$$
\tilde{M}=\bigoplus_{a \in \Sigma} \tilde{\mu}(a)
$$

Then

$$
\ell \leq \rho(\tilde{M}) \mathbb{E} \rho\left(\mu\left(w_{1}\right)\right) \quad\left(=\rho(\tilde{M})+\left(\sum_{a \in \Sigma} \rho(\mu(a)) \times p(a)\right)\right) .
$$

Moreover, if $\tilde{\mu}\left(\Sigma^{+}\right)$is torsion, then the equality holds in (36).

\footnotetext{
${ }^{15}$ Here, $\imath \kappa \mu$ denotes the unique morphism $\mu^{\prime}$ such that $\forall a \in \Sigma, \mu^{\prime}(a)=\imath(\kappa \mu(a))$. Note that in general, $\imath \kappa \mu(w) \neq \imath(\kappa \mu(w))$.
} 
It should be noted that we have by construction

$$
\forall a, \rho(\tilde{M}) \geq \rho(\tilde{\mu}(a))=\mathbb{1}
$$

hence, we can decide if $\tilde{\mu}\left(\Sigma^{+}\right)$is torsion by checking the non trivial inequality $\rho(\tilde{M}) \leq \mathbb{1}$ and by using Algorithm 5.6.

Example 11.2. Consider the semigroup of Example 5.8. Since the normalized semigroup $\tilde{\mu}\left(\Sigma^{+}\right)$is torsion, we have

$$
\ell=\rho(\mu(a)) \times p(a)+\rho(\mu(b)) \times p(b)=1 \times p(b)=p(b) .
$$

Example 11.3. In the same vein, we can build many semigroups whose Lyapunov exponents are immediately obtained. Let

$$
\mu(a)=\left[\begin{array}{lll}
1 & * & * \\
* & * & * \\
* & * & *
\end{array}\right], \mu(b)=\left[\begin{array}{ccc}
2 & \# & \# \\
\# & \# & \# \\
\# & \# & \#
\end{array}\right]
$$

where ${ }^{*}$ stands for arbitrary finite real numbers $\leq 1$ (we allow different values) and \# stands for arbitrary finite real numbers $\leq 2$. Let $\epsilon_{11} \in \mathbb{R}_{\max }^{n \times n}:\left(e_{11}\right)_{i j}=\delta_{i=1, j=1}$ (Kronecker's $\delta$ ). Then, $\psi \tilde{\mu}(a), \psi \tilde{\mu}(b) \geq e_{11}$, and since $e_{11}^{2}=e_{11}, \psi \tilde{\mu}(w)$ is non nilpotent for all $w$. Hence the condition of Theorem 5.7 is satisfied, and we have

$$
\ell=1 \times p(a)+2 \times p(b) .
$$

Proof of Theorem 11.1. For simplicity we shall assume that the alphabet has only two letters ( $\Sigma=\{a, b\}$ ), the extension to the general case being straightforward. We have

$$
\mu(w)=\rho(\mu(a))^{|w|_{a}} \rho(\mu(b))^{|w|_{b}} \tilde{\mu}(w),
$$

hence

$$
(\|\mu(w)\|)^{\frac{1}{|w|}}=\rho(\mu(a))^{\frac{|w|_{a}}{|w|}} \rho(\mu(b))^{\frac{|w|_{b}}{|w|}}(\|\tilde{\mu}(w)\|)^{\frac{1}{|w|}} .
$$

The usual law of large numbers yields:

$$
\lim _{|w| \rightarrow \infty} \rho(\mu(a))^{\frac{|w|_{a}}{|w|}} \rho(\mu(b))^{\frac{|w|_{b}}{|w|}}=\mathbb{E} \rho\left(\mu\left(w_{1}\right)\right) \text { a.s. }
$$

Setting $\tilde{M}=\bigoplus_{a \in \Sigma} \tilde{\mu}(a)$, we obtain

$$
\|\tilde{\mu}(w)\| \leq\left\|\tilde{M}^{|w|}\right\| \leq K \rho(\tilde{M})^{|w|}
$$

for some finite constant $K$. Then, it follows from (38) and (39) that

$$
\lim _{|w| \rightarrow \infty}(\|\mu(w)\|)^{\frac{1}{|w|}} \leq \mathbb{E} \rho\left(\mu\left(w_{1}\right)\right) \rho(\tilde{M}) \text { a.s. }
$$

Moreover, if $\tilde{\mu}\left(\Sigma^{+}\right)$is finite, trivially, $(\| \tilde{\mu}(w)||)^{\frac{1}{|w|}} \rightarrow \mathbb{1}$ as $|w| \rightarrow \infty$, hence, we obtain from $(38),(39)$ the equality in $(40)$.

Acknowledgment The author would like to thank Jean Mairesse for numerous discussions and comments, and particularly, for having suggested the probabilistic application. 


\section{References}

[1] M. Akian, J.P. Quadrat, and M. Viot, Bellman processes, Lect. Notes. in Control and Inf. Sci, no. 199, Springer, June 1994.

[2] F. Baccelli, G. Cohen, G.J. Olsder, and J.P. Quadrat, "Synchronization and linearity", Wiley, 1992.

[3] F. Baccelli and T. Konstantopoulos, Estimates of cycle times in stochastic Petri nets, Proceedings of Workshop on Stochastic Analysis, Rutgers University (I. Karatzas, ed.), Springer, Berlin, 1991.

[4] T.C. Brown, An interesting combinatorial method in the theory of locally finite semigroups, Pacific J. Math. 36 (1971), 285-289.

[5] R. R. Brualdi and H. J. Ryser, "Combinatorial matrix theory", Cambridge University Press, 1991.

[6] G. Cohen, D. Dubois, J.P. Quadrat, and M. Viot, Analyse du comportement périodique des systèmes de production par la théorie des diö̈des, Rapport de recherche 191, INRIA, Le Chesnay, France, 1983.

[7] G. Cohen, P. Moller, J.P. Quadrat, and M. Viot, Algebraic tools for the performance evaluation of discrete event systems, IEEE Proceedings: Special issue on Discrete Event Systems 77 (1989), no. 1.

[8] R.A. Cuninghame-Green, "Minimax Algebra", Lectures notes in Economics and Mathematical Systems, no. 166, Springer, 1979.

[9] A. de Luca, On the Burnside problem for semigroups, In "Mots" (M. Lothaire, ed.), Hermes, 1990.

[10] P. Dudnikov and S. Samborskiu, Endomorphisms of semimodules over semirings with an idempotent operation, Math. in USSR, Izvestija $\mathbf{3 8}$ (1992), no. 1, (translation of Izv. Akad. Nauk SSSR Ser. Mat. 55, 1991).

[11] L. Elsner, C.R. Johnson, and J. Dias da Silva, The Perron root of a weighted geometric mean of nonnegative matrices, Linear Multilinear Algebra 24 (1988), 1-13.

[12] S. Gaubert, Théorie des systèmes linéaires dans les diö̈des, Thèse, École des Mines de Paris, July 1992.

[13] Timed automata and discrete event systems, Proceedings of the ECC93 (Groningen), July 1993.

[14] On rational series in one variable over certain dioids, Rapport de recherche 2162, INRIA, Jan. 1994.

[15] Performance evaluation of $($ max, + ) automata, IEEE Trans. on Automatic Control (1995), to appear (preliminary version: INRIA Report 1922, May 1993).

[16] M. Gondran and M. Minoux, Valeurs propres et vecteurs propres dans les dioïdes et leur interprétation en théorie des graphes, EDF, Bulletin de la Direction des Etudes et Recherches, Serie C, Mathématiques Informatique 2 (1977), 25-41. Graphes et algorithmes, Eyrolles, Paris, 1979, (Engl. transl. "Graphs and Algorithms", Wiley, 1984). Linear algebra in dioids: a survey of recent results, Annals of Discrete Mathematics 19 (1984), 147-164. 
[19] J. Gunawardena ed., "Idempotency", Publications of the Isaac Newton Institute, Cambridge University Press, 1995, to appear.

[20] K. Hashiguchi, Limitedness theorem on finite automata with distance functions, J. Comput. System Sci. 24 (1982), no. 2, 233-244.

[21] Improved limitedness theorems on finite automata with distance functions, Theoret. Comput. Sci. 72 (1990), 27-38.

[22] G. Jacob, La finitude des représentations linéaires des semi-groupes est décidable, J. Algebra 52 (1978), 437-459.

[23] D. Krob, Complete systems of $\mathcal{B}$-rational identities, Theor. Comp. Sci. 89 (1991).

[24] The equality problem for rational series with multiplicities in the tropical semiring is undecidable, Int. J. of Algebra and Comput. 3 (1993).

[25] Some consequences of a Fatou property of the tropical semiring, J. of Pure and Applied Algebra 93 (1994), 231-249.

[26] D. Krob and A. Bonnier-Rigny, A complete system of identities for one letter rational expressions with multiplicities in the tropical semiring, Theoret. Comput. Sci. 134 (1994), 27-50.

[27] H. Leung, On the topological structure of a finitely generated semigroup of matrices, Semigroup Forum 37 (1988), 273-287.

[28] M. Lothaire, "Combinatorics on words", Encyclopedia of Mathematics and its applications, Addison-Wesley, 1983.

[29] J. Mairesse, Products of irreducible random matrices in the $(\max ,+)$ algebra, Rapport de Recherche 1939, INRIA, June 1993, To appear in Adv. Applied Prob.

[30] A graphical approach of the spectral theory in the (max,+) algebra, IEEE Trans. Automatic Control (1995), to appear.

[31] Stabilité des systèmes à événements discrets stochastiques. approche algébrique, Thèse, École Polytechnique, June 1995.

[32] J.P. Mascle, Torsion matrix semigroups and recognizable transductions, In "Automata, Languages and Programming" (L. Kott, editor), Lecture notes in Computer Sciences, 1986, pp. 244-253.

[33] V. Maslov and S. Samborskilu eds., "Idempotent analysis", Adv. in Sov. Math., vol. 13, AMS, RI, 1992.

[34] R. McNaughton and Y. Zalcstein, The Burnside problem for semigroups, J. Algebra 34 (1975), 292-299.

[35] H. Minc, "Nonnegative matrices", Wiley, 1988.

[36] R.D. Nussbaum, Convergence of iterates of a nonlinear operator arising in statistical mechanics, Nonlinearity 4 (1991), 1223 - 1240.

[37] J.E. Pin, "Variétés de langages formels", Masson, Paris, 1984.

[38] M. Plus, A linear system theory for systems subject to synchronization and saturation constraints, Proceedings of the first European Control Conference (Grenoble), July 1991.

[39] C. Reutenauer and H. Straubing, Inversion of matrices over a commutative semiring, J. Algebra 88 (1984), no. 2, 350-360. 
[40] I. Simon, Limited subsets of the free monoid, Proc. of the 19th Annual Symposium on Foundations of Computer Science, IEEE, 1978, pp. 143150 .

[41] Recognizable sets with multiplicities in the tropical semiring, In "Mathematical Foundations of Computer Science" (M.P. Chytil, L. Janiga, and V. Koubek, eds.), Lecture notes Computer Science, vol. 324, Springer, 1988, pp. 107-120.

[42] The nondeterministic complexity of a finite automaton, In "Mots" (M. Lothaire, ed.), Hermes, 1990.

[43] On semigroups of matrices over the tropical semiring, Theor. Infor. and Appl. 28 (1994), no. 3-4, 277-294.

[44] H. Straubing, The Burnside problem for semigroups of matrices, in "Combinatorics on words: progress and perspectives" (L.J. Cummings, ed.), Acad. Press, 1983, pp. 279-295.

INRIA

Domaine de Voluceau

78153 Le Chesnay Cédex

France

Email

Stephane.Gaubert@inria.fr

Received April 1, 1994

and in final form November 27, 1995 